\title{
Fusulinid Foraminifera of the Bashkirian-Moscovian boundary in the eastern Taurides, southern Turkey
}

\author{
ALEKSANDRA V. DZHENCHURAEVA ${ }^{1}$ \& CENGIZ OKUYUCU ${ }^{2}$ \\ ${ }^{1}$ State Agency on Geology and Mineral Resources under the Government of Kyrgyz Republic, KMEGEI, Paleontologic-Stratigraphical \\ Department, Bishkek, Kyrgyzstan \\ ${ }^{2}$ Maden Tetkik ve Arama Genel Müdürlügü, Jeoloji Etütleri Dairesi, 06520 Balgat, Ankara, Turkey (e-mail: okuyucu@mta.gov.tr)
}

\begin{abstract}
Fusulinid faunas of the Pennsylvanian (Bashkirian-Moscovian) deposits of the Siyah Aladag Nappe were investigated from the Kuzuoluk section located in the eastern Taurides. The section is represented by grey, occasionally laminated, algal and fusulinid-rich limestones. The foraminiferal faunas of the Kuzuoluk section are very diverse and abundant which allows identification of the Bashkirian-Moscovian boundary by index-species such as Verella spicata and Aljutovella aljutovica. Other key taxa in the Bashkirian-Moscovian interval include Pseudostaffella grandis and Staffellaeformes staffellaeformis (Bashkirian Stage, upper part of the Lower Substage), Ozawainella pararhomboidalis and Staffellaeformes bona (Bashkirian Stage, Upper Substage), Verella spicata (Bashkirian Stage, Upper Substage) and Aljutovella aljutovica Zones (Moscovian Stage, Lower Substage). The BashkirianMoscovian microfaunas from the Kuzuoluk section can be correlated with assemblages from Tien-Shan, the Southern Urals and the Russian Platform. J. Micropalaeontol. 26(1): 73-85, April 2007.
\end{abstract}

KEYWORDS: Pennsylvanian, Bashkirian-Moscovian boundary, fusulinids, biostratigraphy, Turkey

\section{INTRODUCTION}

Studies concerning the official establishment of Carboniferous stage boundaries started shortly after the VIII International Geological Congress (1975) in Moscow and are still ongoing. The present study is one of several studies undertaken in different regions of the world in order to establish potential boundary marker taxa and stratotype sections for the BashkirianMoscovian boundary. This effort is similar to projects that led to stratotype selection for the lower, middle and upper boundaries of the Carboniferous System by Paproth et al. (1991), Lane et al. (1999), Davydov et al. (1998) and Davydov (2002).

A Task Group to establish a Bashkirian-Moscovian boundary was organized recently and the issues were summarized by Groves (2003). At the XVth International Congress on Carboniferous and Permian Stratigraphy (2003) in Utrecht, the Donbass and the South Urals were identified as the best studied areas for consideration. However, marine sedimentation in this interval occurred also in Tien-Shan (Dzhenchuraeva, 1979, 2001), the Cantabrian Mountains (Villa, 1995) and elsewhere.

The purpose of this study is, first, to give preliminary results for the description and illustration of fusulinid foraminifera in order to determine the Bashkirian-Moscovian boundary from the Kuzuoluk section (Siyah Aladag Nappe) and, secondly, to compare with similar Bashkirian-Moscovian boundaries elsewhere in the Taurides and other regions. The Kuzuoluk section is located to the southeast of Yahyali town $(c .100 \mathrm{~km}$ south of Kayseri) and is one of the most continuous sections of the Siyah Aladag Nappe in this region (Figs 1, 2).

The foraminiferal biostratigraphy of the Siyah Aladag Nappe and its equivalents elsewhere in the Tauride Belt have been studied previously by many researchers (Guvenc, 1965a, b, 1972, 1977; Altiner \& Zaninetti, 1980; Altiner, 1981; Altiner \& Ozgul, 2001; Okuyucu, 2001, 2002, 2003). The most recent studies on Carboniferous biostratigraphy are from the central Taurides (south of Hadim town). In this region, stratigraphical investigation of the Carboniferous (Yaricak Formation) and Permian (Cekic Dagi Formation) deposits of the Aladag Unit were carried out by Ozgul (1997). Foraminiferal faunas and biozones of the Yaricak Formation (Carboniferous) were also presented in great detail by Altiner \& Ozgul (2001).

\section{STRATIGRAPHY}

The Taurides, one of the major units of the Alpine-Himalayan orogenic belt, extend parallel to the Mediterranean Sea coast in southern Anatolia. According to Ozgul $(1976,1984)$, the Taurides can be divided into three parts: the western Taurides, central Taurides and eastern Taurides, based on geological and morphological characteristics. The Tauride Belt is composed of autochthonous and allochthonous units.

One of the allochthonous sequences in the Tauride Belt is the Siyah Aladag Nappe, first defined by Blumenthal (1941) in the eastern Taurides. It is represented mainly by Middle (?)/Upper Devonian to Upper Permian platform-type carbonates and clastics overlain by Lower-Middle Triassic sequences. The Siyah Aladag Nappe and its equivalents are known as the 'Hadim Nappe' (Blumenthal, 1951) or 'Aladag Unit' (Ozgul, 1976) which encompass the whole Taurus Belt.

The fusulinid fauna of the Carboniferous deposits of Siyah Aladag Nappe were examined at the Kuzuoluk section in the eastern Taurides (Fig. 3). This section is located southeast of Yahyali town and is $2 \mathrm{~km}$ northwest of Kuzuoluk village situated in the L34-c3 quadrangle (Yahyali, Kayseri) (Figs 1, 2). The section is represented by grey, occasionally laminated, algal and fusulinid-rich limestones. The Middle Carboniferous deposits are composed of limestones rich in fusulinids and dasycladacean algae (mainly beresellids), with some quartzites present. The foraminiferal fauna recovered proved to be very rich and determination of the age of the rocks was Middle Carboniferous (Viséan) to Lower Permian. Twenty-four samples 
A. V. Dzhenchuraeva \& C. Okuyucu

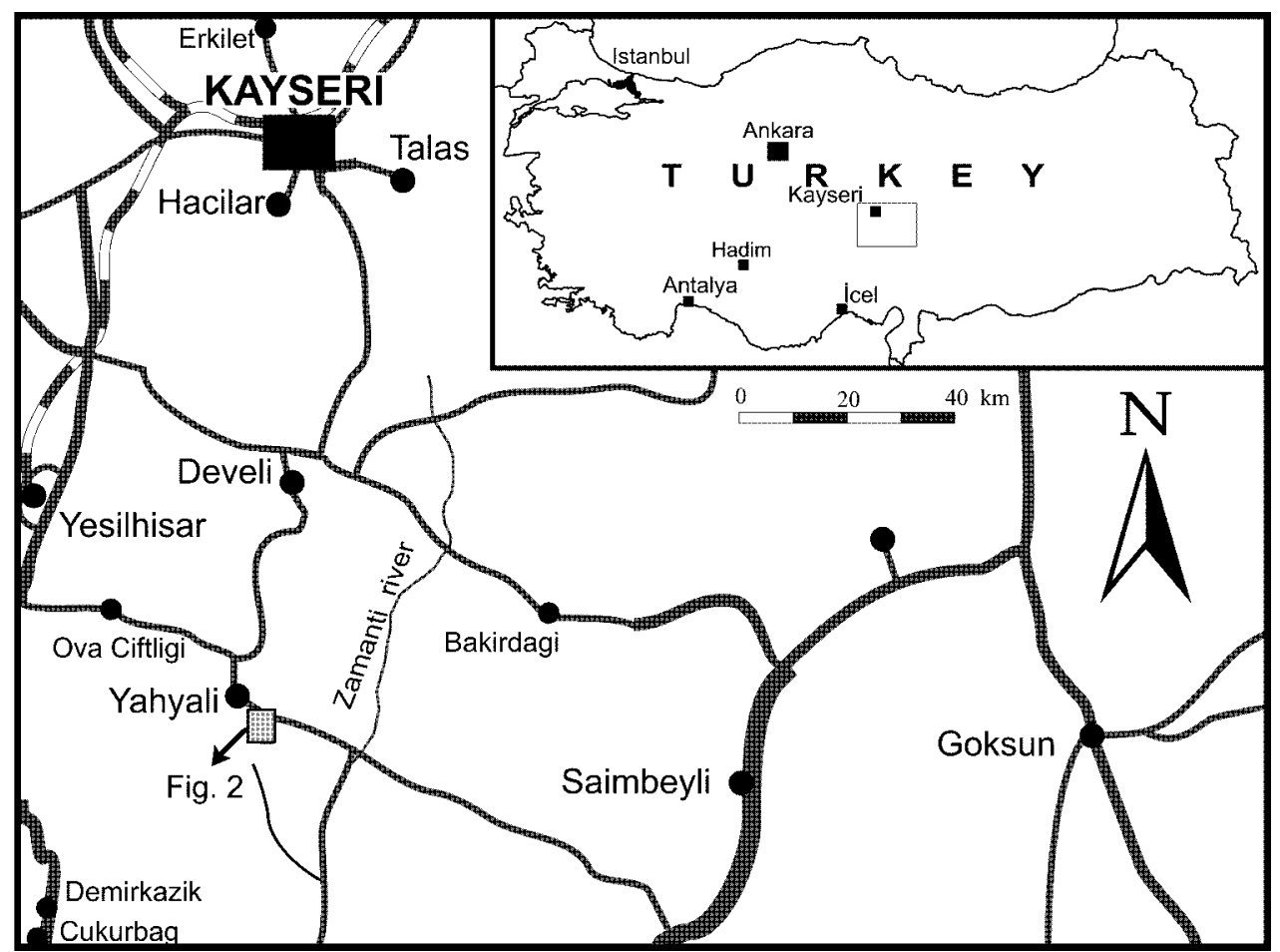

Fig. 1. Location map of the Kuzuoluk section.

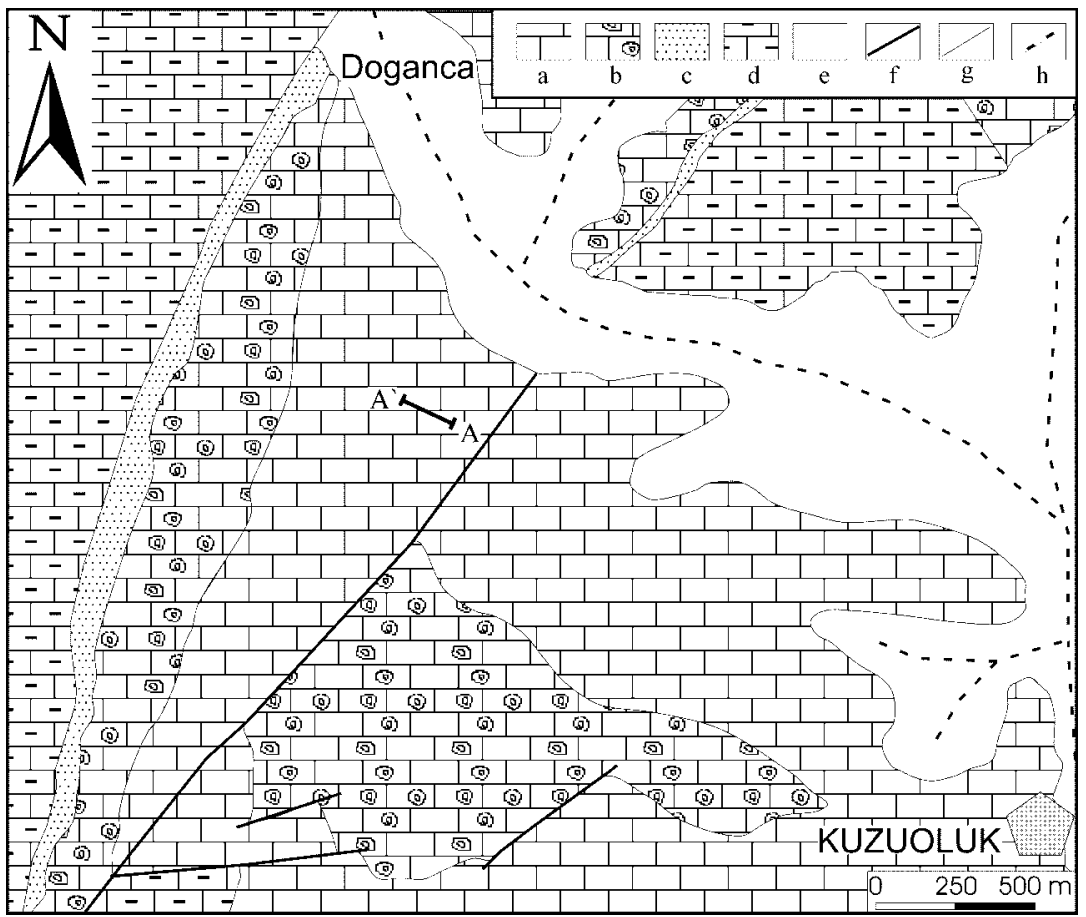

Fig. 2. Geological map of the Kuzuoluk village and its vicinities (simplified after Ayhan \& Lengeranli, 1986) and location of the Kuzuoluk section $\left(\mathrm{A}-\mathrm{A}^{\prime}\right)$. Key: a, Lower and Middle Carboniferous shallow-water limestones with quartz sandstone interlayers; b, Upper Carboniferous-Lower Permian Girvanella-bearing shallow-water limestone (Girvanella Limestone); c, lowest Upper Permian quartz sandstone; d, Upper Permian shallow-water limestones with abundant Mizzia; e, Quaternary deposits; f, fault; g, stratigraphical contact; h, main road.

collected for conodonts from the Kuzuoluk section, including the Bashkirian-Moscovian boundary, proved to be barren. It should be noted that there are some breaks in the Bashkirian strata in the Kuzuoluk section, including the SerpukhovianBashkirian boundary. As a result, three foraminiferal zones (Plectostaffella bogdanovkensis, P. seslavica-Semistaffella 


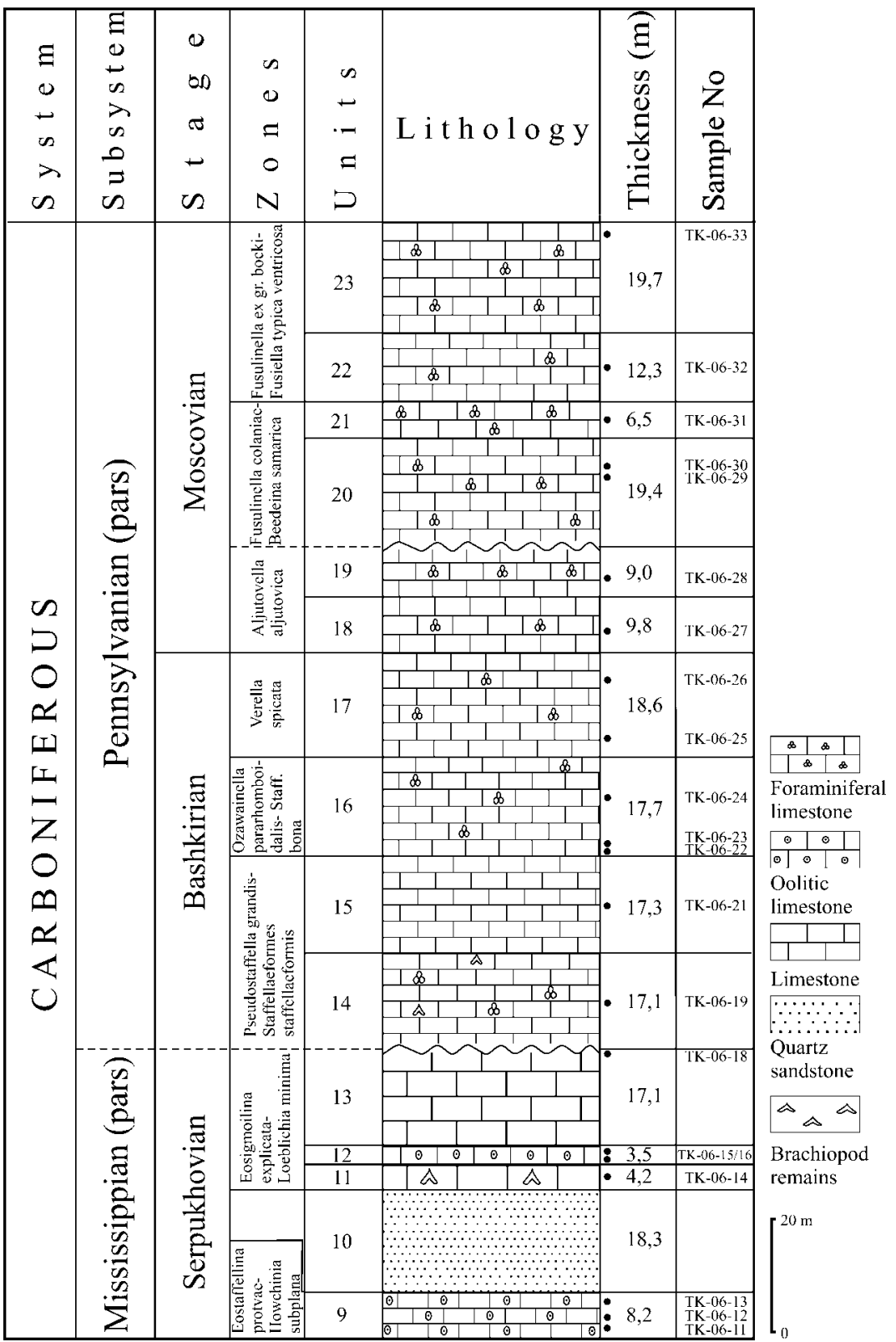

Fig. 3. Stratigraphy of the Kuzuoluk section showing the location of fusulinid-bearing samples and fusulinid zones (Isakova, 2001).

inconstans and P. antiqua) are absent in the studied section. Near the lower and upper Moscovian boundary, the Aljutovella priscoidea and Fusulinella subpulchra Zones respectively are also absent. However, sediments of the upper Bashkirian and lowest Moscovian, including the Bashkirian-Moscovian boundary deposits, are very well exposed (Fig. 3).

\section{Composition and age of the Bashkirian-Moscovian deposits (Units 14-19)}

The aim of this study is to describe and illustrate the fusulinid foraminifera, and stratigraphically characterize the BashkirianMoscovian boundary in the Kuzuoluk section. It is limited to the interval between the Pseudostaffella grandis-Staffellaeformes staffellaeformis (Unit 14) and Aljutovella aljutovica (Unit 19) fusulinid Zones of the Bashkirian-Moscovian interval (biozona- tion follows Isakova, 2001). Investigation of the fusulinids of the Middle Viséan-Serpukhovian and Middle Moscovian-Lower Permian interval is ongoing. The collection is housed in the Natural History Museum of MTA (General Directorate of Mineral Research and Exploration).

Bashkirian Stage, upper part of the Lower Substage (Pseudostaffella grandis-Staffellaeformes staffellaeformis Zone). Unit 14 - sample TK-06-19: Black-dark grey, thick-bedded limestones with brachiopods and foraminifera, including Palaeotextularia sp., Climacammina sp., Eostaffella parastruvei chusovensis Kireeva, E. postmosquensis Kireeva (P1. 1, fig. 7), Millerella umbilicata Kireeva, Plectostaffella bogdanovkensis Reitlinger (Pl. 1, figs 4-5), P. varvariensis (Brazhnikova \& Potievska) (Pl. 1, fig. 1), P. seslavica Rumyantseva (Pl. 1, fig. 6), 

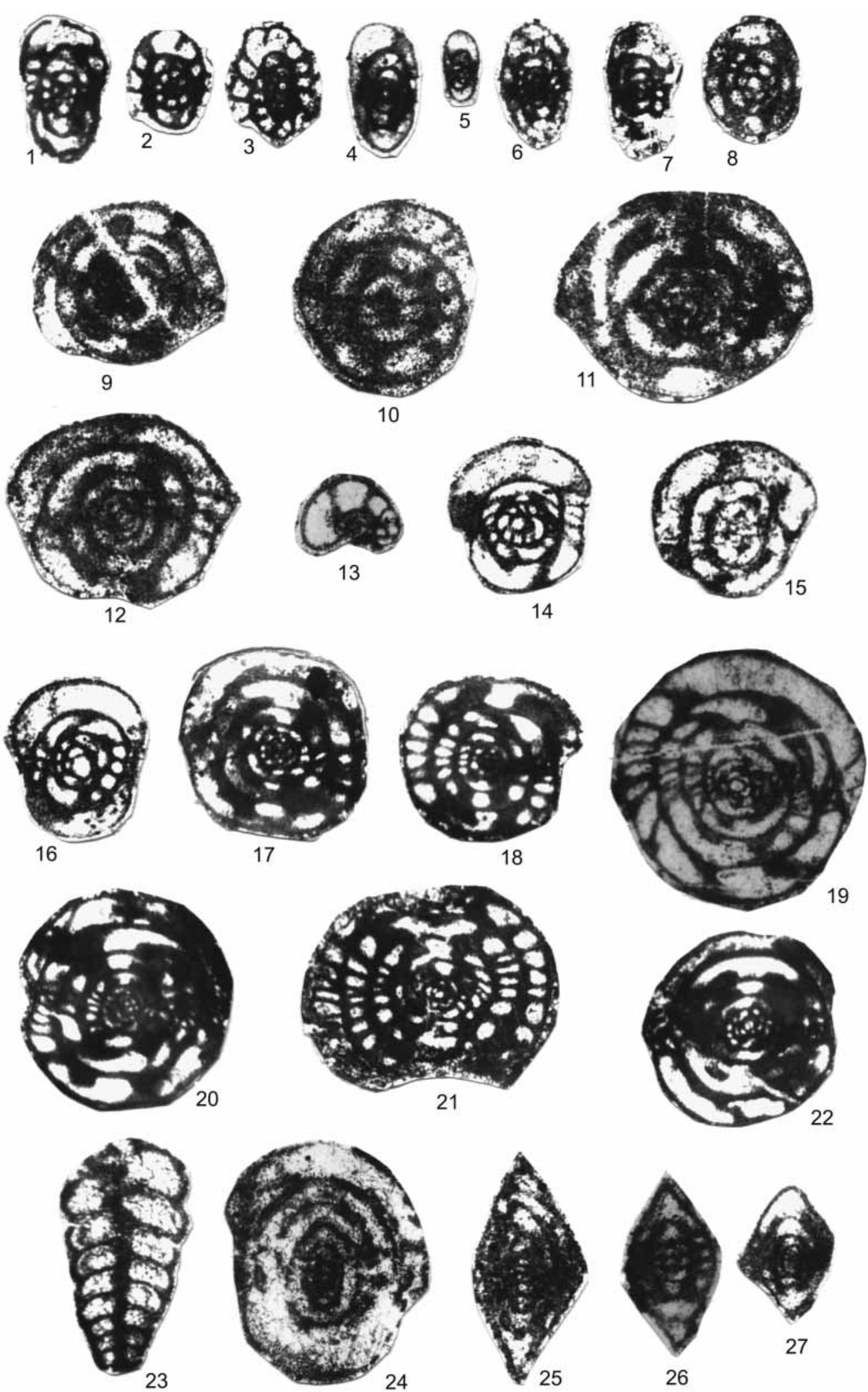

Plate 1. 

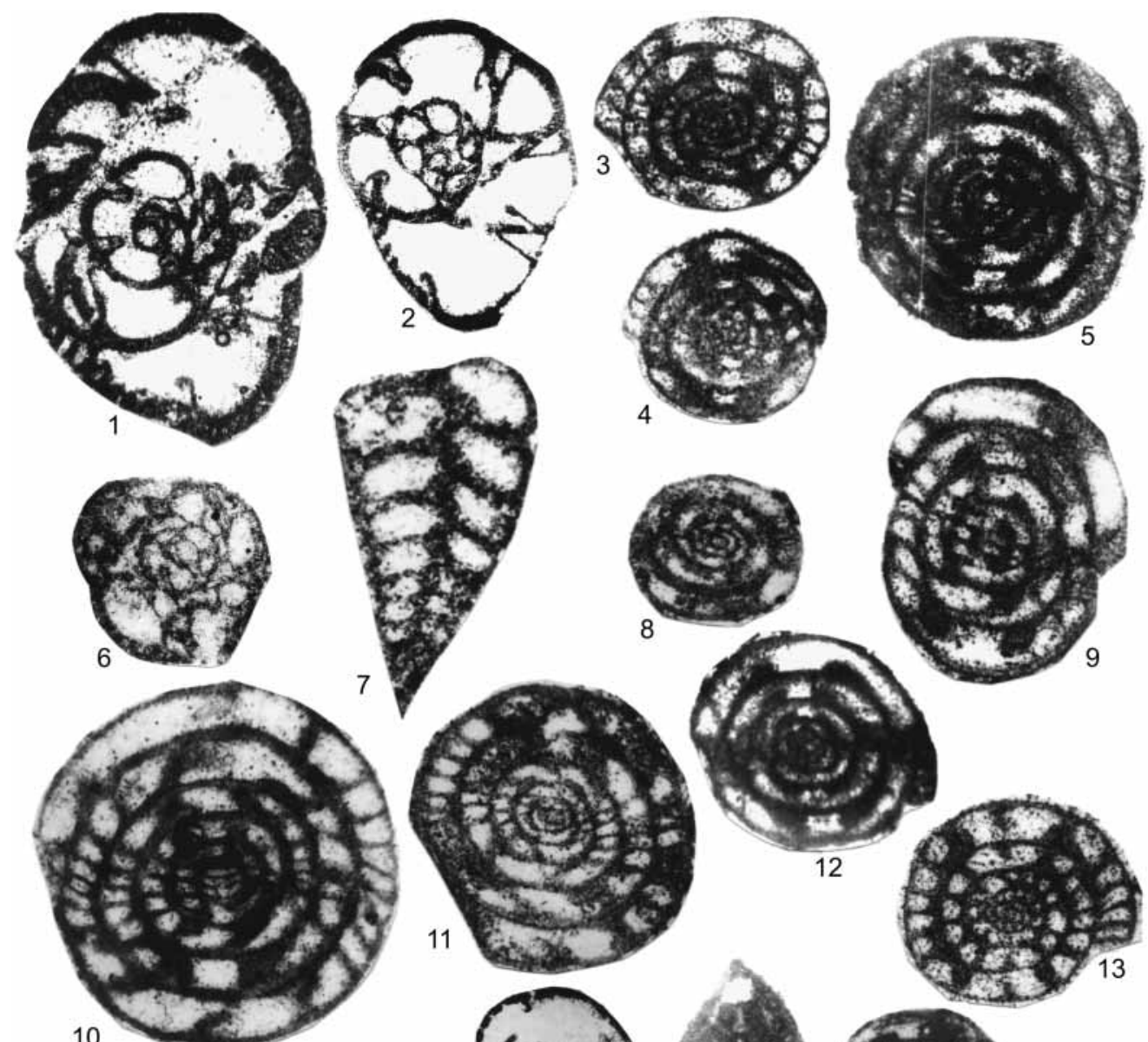

$\left(f_{1}, 2,+3\right.$
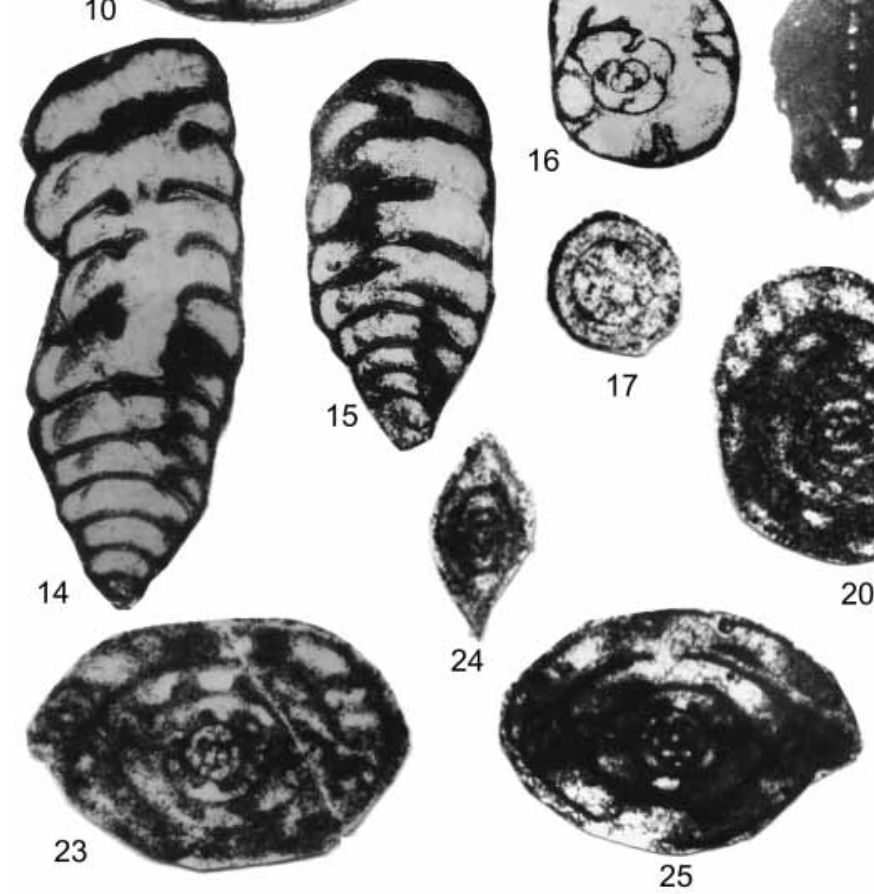

Plate 2.
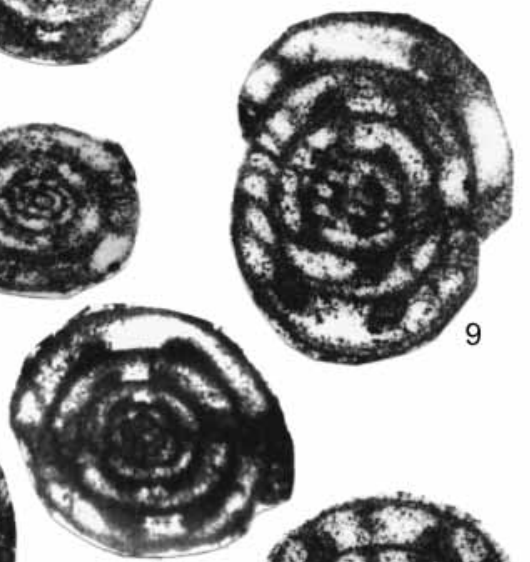


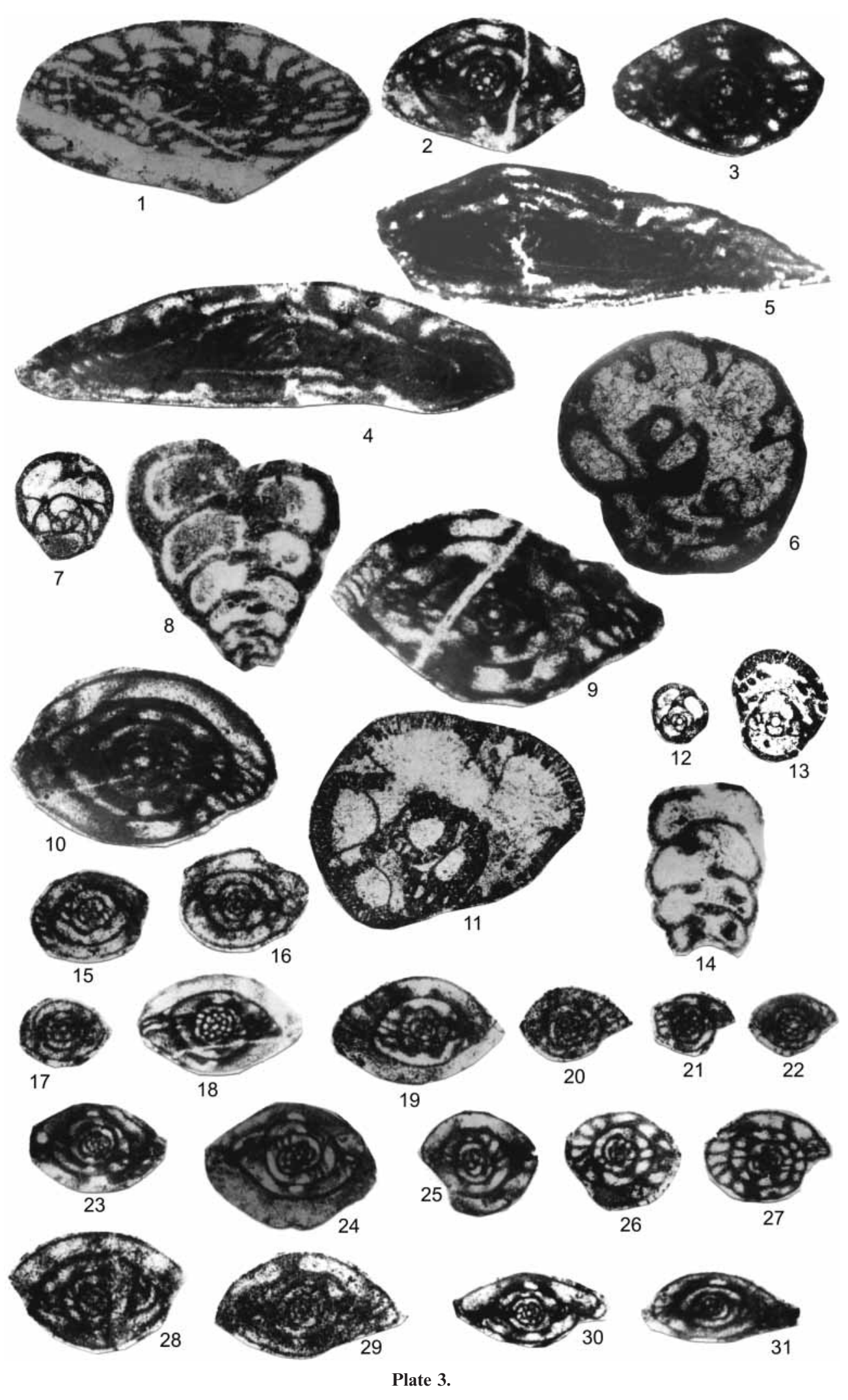




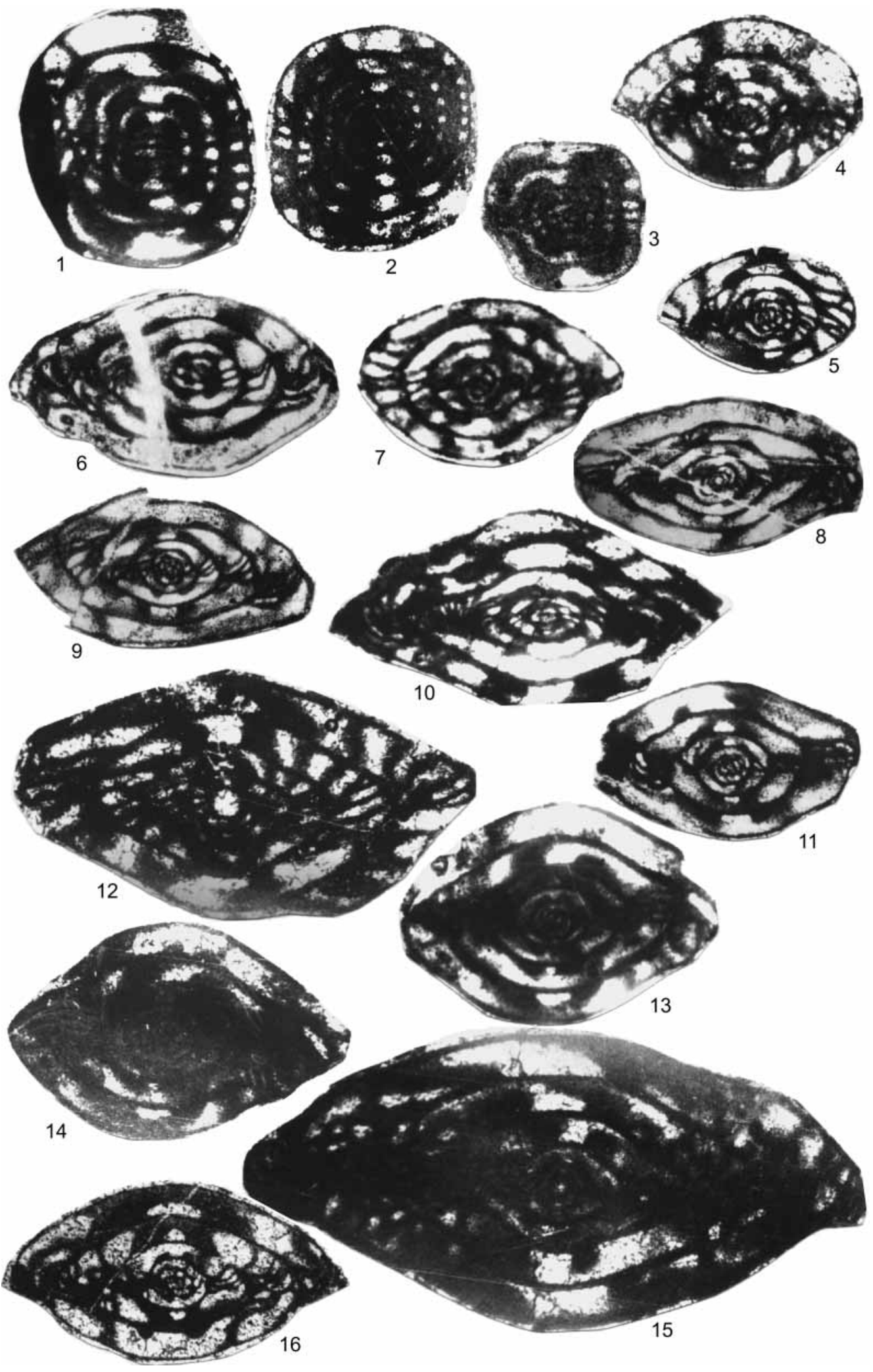

Plate 4. 

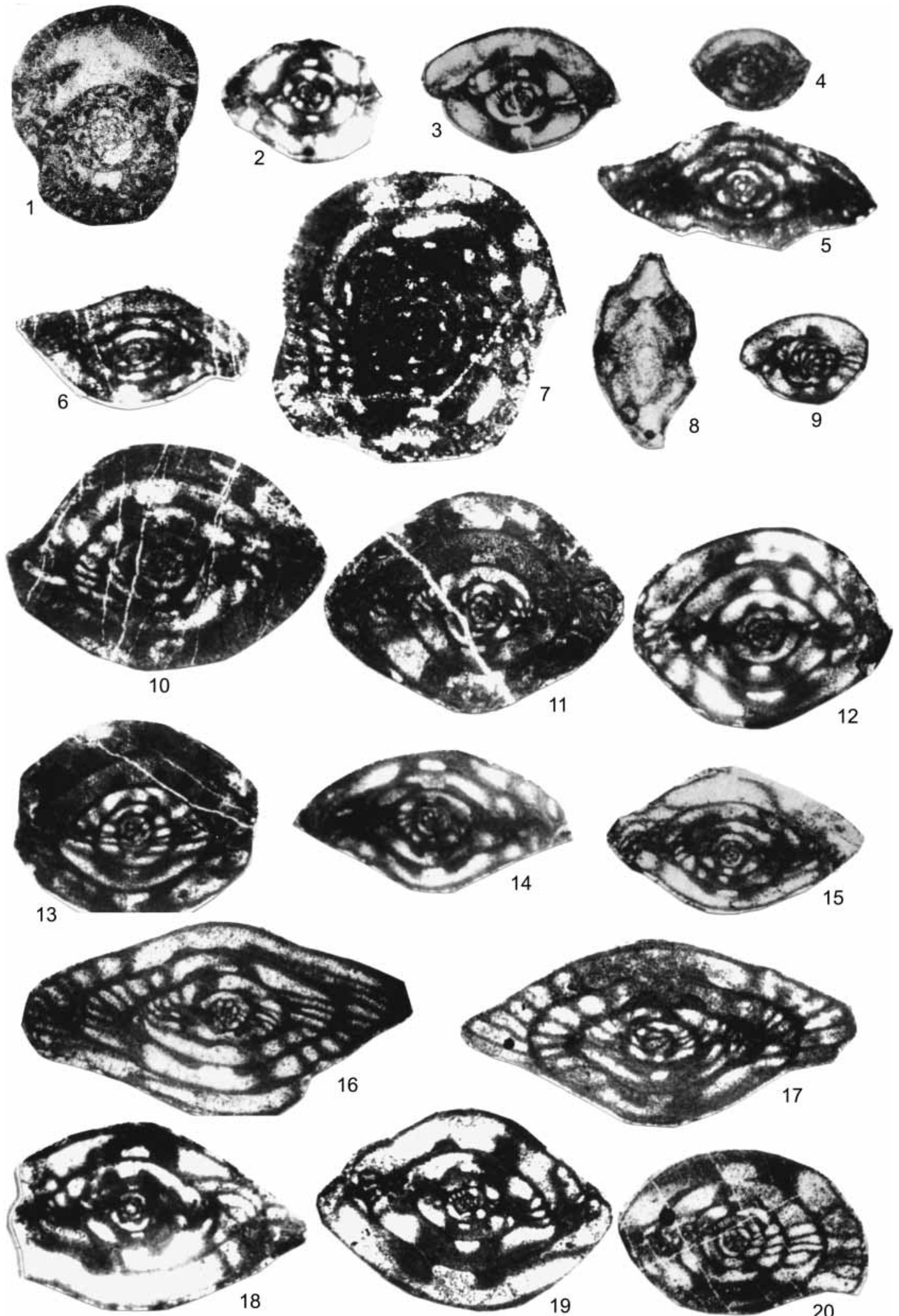

Plate 5.

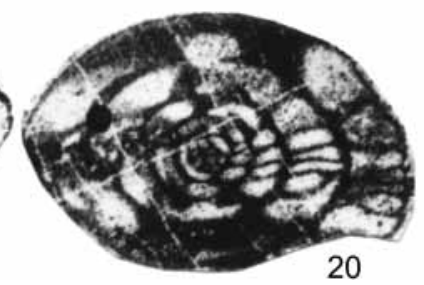


Explanation of Plate 1.

fig. 1. Plectostaffella varvariensis (Brazhnikova \& Potievska); axial section; TK-06-19/5. figs 2-3. Semistaffella minuscularia Reitlinger; axial sections: 2, TK-06-19/7; 3, TK-06-19/4. figs 4-5. Plectostaffella bogdanovkensis Reitlinger; axial sections: 4, TK-06-19/5; 5, TK-06-19/7. fig. 6. Plectostaffella seslavica Rumyantseva; axial section; TK-06-19/6. fig. 7. Eostaffella postmosquensis Kireeva; axial section; TK-06-19/4. fig. 8. Semistaffella variabilis Reitlinger; axial section; TK-06-19/2. figs 9-10. Pseudostaffella antiqua (Dutkevich): 9, axial section; TK-06-19/2(11); 10, subaxial section, TK-09-19/2(3). figs 11-12. Staffellaeformes staffellaeformis (Kireeva), axial sections: 11, TK-06-19/2(1); 12, TK-06-19/2(2). fig. 13. Indeterminate biseriamminid; near axial section; TK-06-21/1. figs 14-16. Pseudostaffella korobezkikh Rauser \& Safonova; axial sections: 14, TK-06-21/5; 15, TK-06-21/6; 16, TK-06-21/5(1). figs 17-18. P. praegorskyi Rauser; axial sections: 17, TK-06-21/2; 18, TK-06-21/1. figs 19-20, 22. P. grandis (Shlykova); axial sections: 19, TK-06-21/2(1); 20, TK-06-21/3; 22, TK-06-21/2. fig. 21. Staffellaeformes staffellaeformis (Kireeva); axial section; TK-06-21/1. fig. 23. Palaeotextularia sp.; longitudinal section; TK-06-22/1. fig. 24. Pseudoendothyra moelleri (Ozawa); subaxial section; TK-06-22/2. figs 25-26. Ozawainella pararhomboidalis Rauser; axial sections: 25, TK-06-22/2(2); 26, TK-06-22/2(1). fig. 27. O. inflata Dzhenchuraeva; axial section; TK-06-22/3. Magnifications: figures 1-19 × 45; figures 20-30 × 31. Figs 1-12 from Unit 14; figs 13-22 from Unit 15; figs 23-27 from Unit 16.

Explanation of Plate 2.

fig. 1. Bradyina cribrostomata Rauser \& Reitlinger; oblique section; TK-06-22/1. fig. 2. B. venusta Reitlinger; sagittal section; TK-06-22/1(1). fig. 3. Staffellaeformes staffellaeformis (Kireeva); subaxial section; TK-06-22/6. fig. 4. Pseudostaffella grandis (Shlykova); axial section; TK-06-22/6. figs 5, 20. P. gorskyi (Dutkevich); axial sections: 5, TK-06-22/3; 20, TK-06-25/3(5). fig. 6. Bradyina sp.; sagittal section; TK-06-23/1. fig. 7. Palaeotextularia eofragilis (Reitlinger); longitudinal section; TK-06-23/1. fig. 8. Pseudostaffella antiqua (Dutkevich); axial section; TK-06-23/2(1). fig. 9. P. praegorskyi Rauser; subaxial section; TK-06-23/2(2). figs 10-11. P. grandis (Shlykova); axial sections: 10, TK-06-23/3(1); 11, TK-06-23/3(2). figs 12-13. Staffellaeformes bona Grozdilova \& Lebedeva: 12, axial section; TK-06-23/3(3); 13, sagittal section; TK-06-23/2(3). figs 14-15. Deckerella sp.; longitudinal sections: 14, TK-06-24/1; 15, TK-06-24/2. fig. 16. Bradyina sp.; sagittal section; TK-06-24/3. fig. 17. Bituberitina devonica Poyarkov; longitudinal section; TK-06-25/3(1). fig. 18. Ozawainella inflata Dzhenchuraeva; axial section; TK-06-25/3(2). figs 19, 21. Staffellaeformes bona (Grozdilova \& Lebedeva); axial sections: 19, TK-06-25/4; 21, TK-06-25/1. fig. 22. Pseudostaffella paracompressa Safonova; axial section; TK-06-25/3(1). fig. 23. Staffellaeformes staffellaeformis (Kireeva); axial section; TK-06-25/3(2). fig. 24. Ozawainella concinnae Dzhenchuraeva; axial section; TK-06-25/3(3). fig. 25. Profusulinella parva Lee \& Chen; axial section; TK-06-25/3(4). fig. 26. P. parva robusta Rauser \& Belyaev; axial section; TK-06-25/3(5). Magnifications: all figures $\times 31$ except 1,2, 6, 14, 15 and $16 \times 15.4$. Figs $1-16$ from Unit 16; figs 17-26 from Unit 17 .

Explanation of Plate 3.

figs 1, 9. Aljutovella tikhonovichi Rauser: 1, near axial section; TK-06-25/5; 9, axial section; TK-06-26-/1(3). figs 2-3. Profusulinella convoluta Lee \& Chen; axial sections: 2, TK-06-25/4; 3, TK-06-25/2. figs 4-5. Verella spicata Dalmatskaya; axial sections: 4, TK-06-25/7; 5, TK-06-25/8. figs 6, 11. Bradyina sp. (aff. cribrostomata Rauser \& Reitlinger); tangential sections: 6, TK-06-26/2(2); 11, TK-06-26/1(1). figs 7, 13. B. venusta Reitlinger; axial sections: 7, TK-06-27/1(2); 13, TK-06-27/3(1). fig. 8. Textularia panderosa Reitlinger; longitudinal section; TK-06-26/1(2). fig. 10. Profusulinella parva Lee \& Chen; axial section; TK-06-26/2(1). fig. 12. Bradyina minima Reitlinger; central oblique section; TK-06-27/3(8). fig. 14. Climacammina sp.; longitudinal section; TK-06-27/3(10). figs 15-17. Schubertella obscura Lee \& Chen; axial sections: 15, TK-06-27/1(5); 16, TK-06-27/1(7); 17, TK-06-27/1(1). figs 18-19, 23-24. S. gracilis kulensis Dzhenchuraeva; axial sections: 18, TK-06-27/3(4); 19, TK-06-27/1(15); 23, TK-06-27/3(4); 24, TK-06-27/8(3). figs 20-22. S. obscura procera Rauser: 20, subaxial section; TK-06-27/1(10); 21, subaxial section; TK-06-27/1(14); 22, axial section; TK-06-27/1(9). figs 25-27. S. subglobulosa Dzhenchuraeva; axial sections: 25, TK-06-27/8; 26, TK-06-27/3(4); 27, TK-06-27/1(10). figs 28-29. S. magna Lee \& Chen; axial sections: 28, TK-06-27/1(4); 29, TK-06-27/1(2). figs 30-31. S. stricta Dzhenchuraeva; axial sections: 30, TK-06-27/3(5); 31, TK-06-27/3(6). Magnifications: all figures $\times 31$ except 6, 7, 11, 12 and $13 \times 15.4$. Figs 1-6, 8-11 from Unit 17; figs 7, 12-31 from Unit 18 .

Explanation of Plate 4.

fig. 1. Neostaffella nibelensis (Rauser); axial section; TK-06-27/3. fig. 2. N. pseudoquadrata (Manukalova); axial section; TK-06-27/3(2). fig. 3. N. subquadrata (Grozdilova \& Lebedeva); axial section; TK-06-27/3(1). fig. 4. Profusulinella parva Lee \& Chen; axial section; TK-06-27/1. fig. 5. P. convoluta (Lee \& Chen); axial section; TK-06-27/2. figs 6-7. Ovatella subovata (Safonova); axial section: 6, TK-06-27/3; 7, TK-06-27/4. figs 8-9. O. nytvica (Safonova); axial sections: 8, TK-06-27/1; 9, TK-06-27/1(2). fig. 10. Profusulinella rhomboides (Lee \& Chen); axial section; TK-06-27/5. fig. 11. Ovatella ovata penduelesensis (Ginkel); axial section; TK-06-27/1(3). fig. 12. Profusulinella pararhomboides Rauser \& Belyaev; axial section; TK-06-27/5. figs 13-14. P. paratimanica Rauser; axial sections: 13, TK-06-27/6(2); 14, TK-06-27/6(1). fig. 15. Ovatella constans (Safonova); axial section; TK-06-27/2. fig. 16. Aljutovella aljutovica Rauser; axial section; TK-06-27/3. Magnifications: all figures $\times 31$. Figs $1-16$ from Unit 18 .

Explanation of Plate 5.

fig. 1. Bradyina sp. (aff. cribrostomata Rauser \& Reitlinger); subaxial section; TK-06-28/1(2). figs 2-3. Schubertella gracilis kulensis Dzhenchuraeva; axial sections: 2, TK-06-28/1; 3, TK-06-28/3. figs 4, 9. S. obscura Lee \& Chen; axial section: 4, TK-06-28/2; 9, TK-06-28/4. figs 5-6. S. stricta Dzhenchuraeva; axial section: 5, TK-06-28/2(1); 6, TK-06-28/3(20). fig. 7. Neostaffella umbilicata (Putrya \& Leontovich); subaxial section; TK-06-28/2. fig. 8. Pseudoendothyra timanica (Rauser); subaxial section; TK-06-28/3(1). figs 10-11. Depratina prisca (Deprat); axial sections: 10, TK-06-28/7(10); 11, TK-06-28/7(1). figs 12-13. D. timanica (Kireeva); axial sections: 12, TK-06-28/7; 13, TK-06-28/1(1). figs 14-15. Profusulinella minima Dzhenchuraeva; axial sections: 14, TK-06-28/2; 15, TK-06-28/9. figs 16-17. P. rhomboides (Lee \& Chen); subaxial sections: 16, TK-06-28/3(1); 17, TK-06-28/8(6). fig. 18. Ovatella subovata (Safonova); axial section; TK-06-28/3(7). figs 19-20. Aljutovella subaliutovica Safonova: 19, axial section; TK-06-28/1; 20, subaxial section; TK-06-28/1(2). Magnifications: all figures $\times 31$ except $1 \times 15.5$. Figs $1-20$ from Unit 19 . 
Semistaffella variabilis Reitlinger (Pl. 1, fig. 8), S. minuscularia Reitlinger (Pl. 1, figs 2-3), Pseudostaffella antiqua (Dutkevich) (Pl. 1, figs 9-10) and Staffellaeformes staffellaeformis (Kireeva) (Pl. 1, figs 11-12).

Unit 15 - sample TK-06-21: Grey, thick-bedded limestones with indeterminate biseriamminids (P1. 1, fig. 13), Pseudoendothyra bradyi (Moeller), P. ex gr. moelleri (Ozawa), Pseudostaffella grandis (Shlykova) (P1. 1, figs 19-20, 22), P. korobezkikh Rauser \& Safonova (P1. 1, figs 14-16), P. praegorskyi Rauser (Pl. 1, figs 17-18), Schubertella sp. and Staffellaeformes staffellaeformis (Kireeva) (Pl. 1, fig. 21).

Upper Substage (Ozawainella pararhomboidalis-Staffellaeformes bona Zone). Unit 16 - sample TK-06-22: Grey, thick-bedded algal limestones with gastropods and the foraminifera Bradyina cribrostomata Rauser \& Reitlinger (Pl. 2, fig. 1), B. venusta Reitlinger (Pl. 2, fig. 2), Palaeotextularia sp. (Pl. 1, fig. 23), Pseudoendothyra moelleri (Ozawa) (Pl. 1, fig. 24), Semistaffella sp. (aff. inconstans Reitlinger), Pseudostaffella grandis (Shlykova) (Pl. 2, fig. 4), P. gorskyi (Dutkevich) (Pl. 2, fig. 5), Ozawainella pararhomboidalis (Manukalova) (Pl. 1, figs 25-26), O. inflata Dzhenchuraeva (P1. 1, fig. 27), Staffellaeformes staffellaeformis (Kireeva) (Pl. 2, fig. 3), and the algae Ungdarella sp. and Beresella sp.

Sample TK-06-23: Foraminifera Bradyina sp. (P1. 2, fig. 6), Semistaffella inconstans Reitlinger, Palaeotextularia eofragilis (Reitlinger) (P1. 2, fig. 7), Pseudostaffella antiqua (Dutkevich) (Pl. 2, fig. 8), P. grandis (Shlykova) (Pl. 2, figs 10-11), P. praegorskyi Rauser (Pl. 2, fig. 9) and Staffellaeformes bona (Grozdilova \& Lebedeva) (P1. 2, figs 12-13).

Sample TK-06-24: Foraminifera Bradyina sp. (Pl. 2, fig. 16) and Deckerella sp. (Pl. 2, figs 14-15) and the algae Beresella sp.

Verella spicata Zone. Unit 17 - sample TK-06-25: Grey, thickbedded limestones with the foraminifera Bituberitina devonica Poyarkov (Pl. 2, fig. 17), Climacammina sp., Eostaffella acuta Rauser, Bradyina sp., Pseudostaffella ex gr. antiqua (Dutkevich), P. paracompressa Safonova (P1. 2, fig. 22), Staffellaeformes staffellaeformis (Kireeva) (Pl. 2, fig. 23), S. bona (Grozdilova \& Lebedeva) (Pl. 2, figs 19, 21), Profusulinella parva Lee \& Chen (P1. 2, fig. 25), P. parva robusta Rauser \& Belyaev (Pl. 2, fig. 26), P. convoluta Lee \& Chen (Pl. 3, figs 2-3), Aljutovella tikhonovichi Rauser (Pl. 3, fig. 1), Ozawainella inflata Dzhenchuraeva (Pl. 2, fig. 18), O. concinnae Dzhenchuraeva (P1. 2, fig. 24), Verella spicata Dalmatskaya (P1. 3, figs 4-5) and Verella sp.

Sample TK-06-26: Foraminifera Textularia panderosa Reitlinger (Pl. 3, fig. 8), Bradyina sp. (aff. cribrostomata Rauser \& Reitlinger) (P1. 3, figs 6, 11), Eostaffella acuta Rauser, Ozawainella sp., Profusulinella parva Lee \& Chen (Pl. 3, fig. 10), P. ex gr. pararhomboides Rauser \& Belyaev and Aljutovella tikhonovichi Rauser (Pl. 3, fig. 9).

Moscovian Stage, Lower Substage (Aljutovella aljutovica Zone). Unit 18 - sample TK-06-27: Grey, thick-bedded algal limestone with the foraminifera Bradyina sp. (aff. cribrostomata Rauser \&
Reitlinger), B. minima Reitlinger (P1. 3, fig. 12), B. venusta Reitlinger (Pl. 3, figs 7, 13), Climacammina sp. (Pl. 3, fig. 14), Schubertella subglobulosa Dzhenchuraeva (Pl. 3, figs 25-27), S. gracilis kulensis Dzhenchuraeva (P1. 3, figs 18-19, 23-24), S. magna Lee \& Chen (P1. 3, figs 28-29), S. obscura Lee \& Chen (Pl. 3, figs 15-17), S. obscura procera Rauser (P1. 3, figs 20-22), $S$. stricta Dzhenchuraeva (Pl. 3, figs 30-31), Staffellaeformes bona (Grozdilova \& Lebedeva), Ovatella subovata (Safonova) (Pl. 4, figs 6-7), O. nytvica (Safonova) (Pl. 4, figs 8-9), O. ovata penduelesensis (Ginkel) (Pl. 4, fig. 11), O. constans (Safonova) (Pl. 4, fig. 15), Profusulinella pararhomboides Rauser \& Belyaev (Pl. 4, fig. 12), P. paratimanica Rauser (Pl. 4, figs 13-14), $P$. rhomboides Lee \& Chen (Pl. 4, fig. 10), P. parva Lee \& Chen (Pl. 4, fig. 4), P. convoluta (Lee \& Chen) (Pl. 4, fig. 5), Ozawainella kurakhovensis Manukalova, Eofusulina sp., Aljutovella aljutovica Rauser (P1. 4, fig. 16), Neostaffella subquadrata (Grozdilova \& Lebedeva) (Pl. 4, fig. 3), N. pseudoquadrata (Manukalova) (Pl. 4, fig. 2) and $N$. nibelensis Rauser (Pl. 4, fig. 1).

Unit 19 - sample TK-06-28: Grey to dark grey thick-bedded limestones with the foraminifera Bradyina sp. (aff. cribrostomata Rauser \& Reitlinger) (Pl. 5, fig. 1), Pseudoendothyra ex gr. bradyi (Moeller), P. ex gr. moelleri (Ozawa), P. timanica Rauser (P1. 5, fig. 8), Schubertella gracilis kulensis Dzhenchuraeva (Pl. 5, figs 2-3), S. acuta Rauser, S. obscura Lee \& Chen (P1. 5, figs 4, 9), S. stricta Dzhenchuraeva (P1. 5, figs 5-6), Neostaffella umbilicata (Putrya \& Leontovich) (Pl. 5, fig. 7), Depratina prisca (Deprat) (Pl. 5, figs 10-11), D. paratimanica (Rauser \& Belyaev), D. timanica (Kireeva) (Pl. 5, figs 12-13), Profusulinella minima Dzhenchuraeva (Pl. 5, figs 14-15), P. fittsi (Thompson), $P$. rhomboides Lee \& Chen (P1. 5, figs 16-17), Ovatella subovata (Safonova) (Pl. 5, fig. 18), Aljutovella subaljutovica Safonova (Pl. 5, figs 19-20) and Fusulinella ? sp. (aff. ginkeli Villa).

\section{BIOSTRATIGRAPHY}

The Verella spicata and Aljutovella aljutovica fusulinid Zones and their associated fauna were recorded from the BashkirianMoscovian boundary beds (Fig. 4). The characteristic assemblages of the Verella spicata Zone include: Pseudostaffella gorskyi (Dutkevich), Staffellaeformes staffellaeformis (Kireeva), S. bona (Grozdilova \& Lebedeva), Profusulinella parva Lee \& Chen, $P$. ex gr. rhomboides Lee \& Chen, Aljutovella tikhonovichi Rauser, Verella spicata Dalmatskaya and Verella sp.; while the Aljutovella aljutovica Zone includes: Profusulinella pararhomboides Rauser \& Belyaev, P. rhomboides Lee \& Chen, Ovatella subovata (Safonova), O. nytvica (Safonova), Depratina prisca (Deprat), D. paratimanica (Rauser \& Belyaev), Aljutovella aljutovica Rauser, A. aff. intermixta Safonova and Eofusulina sp. (Fig. 4).

The identification of several fusulinid species permits correlation of this section with other global regions (Fig. 5). Among these species, forms such as Verella spicata and Aljutovella aljutovica allow identification of the Bashkirian-Moscovian boundary which, in Kuzuoluk, is located between Units 17 and 18. The base of Moscovian is characterized by the first occurrence of the zonal index Aljutovella aljutovica (Fig. 4).

\section{CORRELATION}

Fusulinid zones and their characteristic index species from Kuzuoluk allow correlation and characterization of the 
Carboniferous fusulinids, southern Turkey

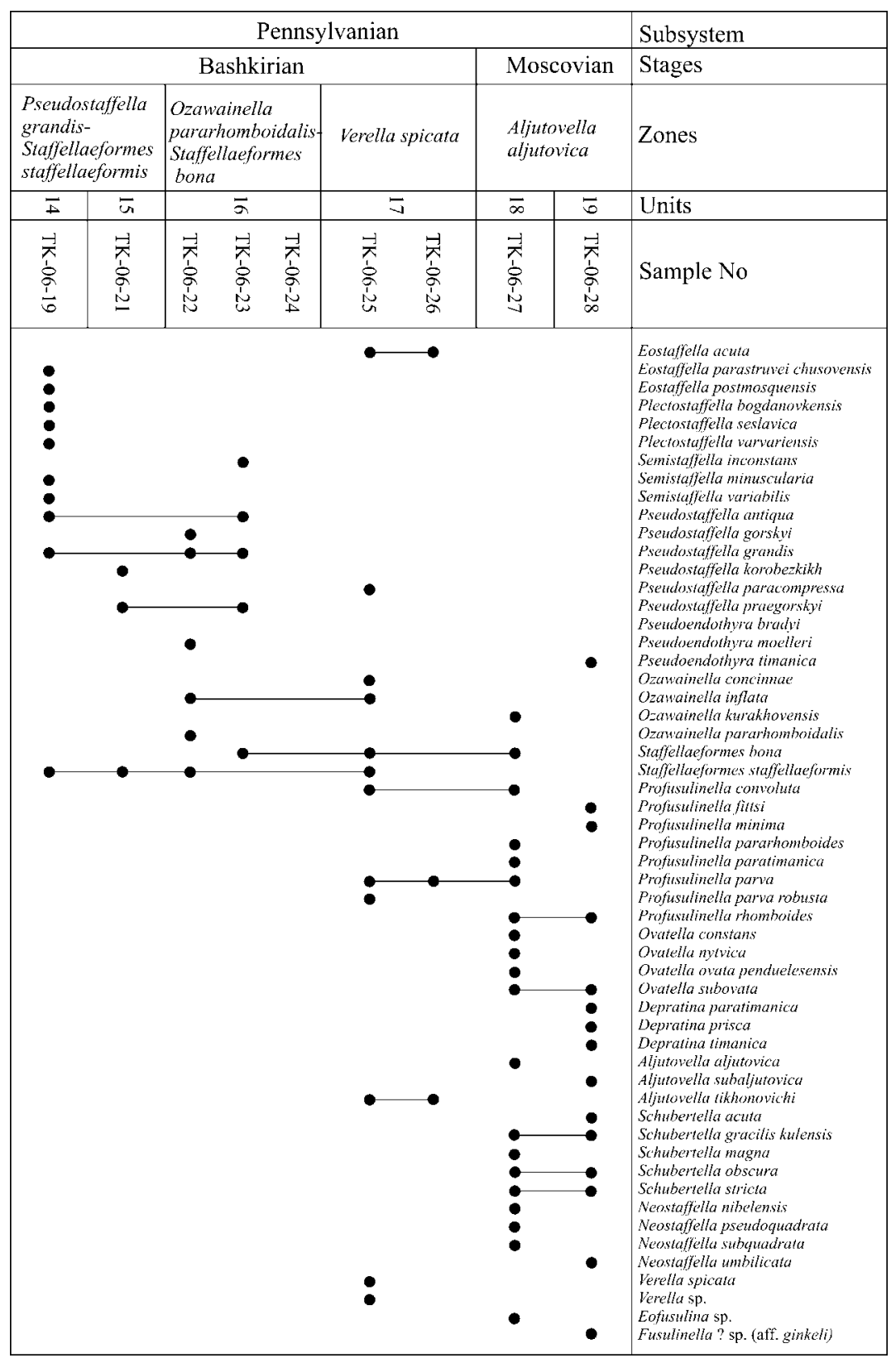

Fig. 4. Stratigraphical distribution of the most important fusulinid fauna in the Kuzuoluk section of the Bashkirian-Moscovian boundary from Units 14-19.

Bashkirian-Moscovian boundary with the central Taurides, Tien-Shan, Southern Urals and Russian Platform (Fig. 5).

The Verella spicata Zone at Kuzuoluk can be correlated partly with the Profusulinella Zone in the central Taurides, corresponding to the final two horizons of the Bashkirian stage of the Yaricak Formation (Fig. 5). The following species are common to both areas: Staffellaeformes staffellaeformis, S. bona, Profusulinella parva, Pseudostaffella gorskyi, and $P$. ex gr. rhomboides. The main faunal difference between the Verella spicata Zone (Kuzuoluk section) and the Profusulinella Zone (Yaricak Formation) is the presence of the index-species Verella spicata in the former section.
The Verella spicata Zone (Kuzuoluk) can be correlated with the Verella spicata-Profusulinella rhombiformis-Aljutovella tikhonovichi Zone (Melekesian Horizon) of the Russian Platform by the presence of index-taxa such as Verella spicata, Aljutovella tikhonovichi and Profusulinella ex gr. rhomboides (Fig. 5). The fusulinid fauna of the lower Moscovian Aljutovella aljutovica Zone (Vereian Horizon) can be correlated by common index-species such as Aljutovella aljutovica, as well as Schubertella acuta, S. obscura, Depratina prisca, Ovatella ovata and Neostaffella subquadrata groups. No species belonging to the Schubertella pauciseptata group in the Kuzuoluk section were recovered. 
A. V. Dzhenchuraeva \& C. Okuyucu

\begin{tabular}{|c|c|c|c|c|c|}
\hline 崩 & $\begin{array}{c}\text { EASTERN } \\
\text { TAURIDES } \\
\text { (Southern Turkey) } \\
\text { This study } \\
\text { Fusulinid zones } \\
\end{array}$ & $\begin{array}{c}\text { CENTRAL } \\
\text { TAURIDES } \\
\text { (Southern Turkey) } \\
\text { Altiner \& Ozgul, 2001 } \\
\text { Fusulinid zones } \\
\end{array}$ & $\begin{array}{c}\text { RUSSIAN } \\
\text { PLATFORM } \\
\text { Solovieva, 1986; } \\
\text { Isakova, 1998, 2001 } \\
\\
\text { Fusulinid zones } \\
\end{array}$ & $\begin{array}{c}\text { SOUTHERN AND } \\
\text { NORTHERN } \\
\text { URALS } \\
\text { Kulagine } \text { et al. } 2001 ; \\
\text { Ivanova, 2000, 2002 } \\
\text { Fusulinid zones } \\
\end{array}$ & $\begin{array}{c}\text { CENTRAL } \\
\text { ASIA } \\
\text { Solovieva, 1977; } \\
\text { Dzhcnchuracva, 1979, } \\
\text { 1997, 2000; } \\
\text { Rumyantseva, 1974 } \\
\text { Fusulinid zones } \\
\end{array}$ \\
\hline 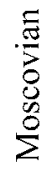 & $\begin{array}{l}\text { Aljutovella } \\
\text { aljutovica }\end{array}$ & $\begin{array}{c}\text { Eostaffella mutabilis- } \\
\text { Profusulinella prisca- } \\
\text { Eofusulina } \\
\text { (Paraeofusulina) }\end{array}$ & $\begin{array}{l}\text { Aljutovella } \\
\text { aljutovica, } \\
\text { Schubertella } \\
\text { pauciseptata }\end{array}$ & $\begin{array}{c}\text { Aljutovella } \\
\text { aljutovica, } \\
\text { Profusulinella } \\
\text { prisca }\end{array}$ & $\begin{array}{c}\text { Aljutovella } \\
\text { aljutovica }\end{array}$ \\
\hline : & $\begin{array}{l}\text { Verella } \\
\text { spicata }\end{array}$ & Profusulinella & $\begin{array}{c}\text { Verella } \\
\text { spicata, } \\
\text { Aljutovella } \\
\text { tikhonovichi }\end{array}$ & $\begin{array}{c}\text { Verella spicata } \\
\text { (for Middle and } \\
\text { Northern Urals), } \\
\text { Aljutovella } \\
\text { tikhonovichi }\end{array}$ & $\begin{array}{l}\text { Verella } \\
\text { spicata }\end{array}$ \\
\hline
\end{tabular}

Fig. 5. Correlation of the described biozones from the Bashkirian-Moscovian boundary strata in the Kuzuoluk section (eastern Taurides) with different regions of Eurasia (Rumyantseva, 1974; Solovieva, 1977, 1986; Dzhenchuraeva, 1979, 1997, 2000; Isakova, 1998, 2001; Ivanova, 2000, 2002; Altiner \& Ozgul, 2001; Kulagine et al., 2001).

The Verella spicata Zone fusulinids at Kuzuoluk can also be correlated with taxa from the Aljutovella tikhonovichi Zone in the Southern Urals (Asatausky Horizon) by species such as Profusulinella ex gr. rhomboides, P. parva, Staffellaeformes staffellaeformis, Pseudostaffella gorskyi, Eostaffella acuta, Aljutovella tikhonovichi and Verella spicata (Fig. 5). The Aljutovella aljutovica Zone at Kuzuoluk can be correlated with the Aljutovella aljutovica-Profusulinella prisca Zone (the Vereian Horizon) using Neostaffella subquadrata, Schubertella gracilis and Eofusulina triangula.

The latest Bashkirian Verella spicata Zone at Kuzuoluk section can be correlated with faunas from the Verella spicata Zone of Central Asia (upper part of the Devatash Horizon) by Staffellaeformes staffellaeformis, S. bona, Profusulinella parva, $P$. ex gr. rhomboides, Aljutovella tikhonovichi and Verella spicata (Fig. 5). Finally, the lower Moscovian Aljutovella aljutovica Zone at Kuzuoluk can also be correlated with the Aljutovella aljutovica Zone in Central Asia based on the presence of Schubertella subglobulosa, S. gracilis kulensis, S. magna, S. acuta, Profusulinella rhomboides, Ovatella ovata, Depratina ex gr. prisca, Aljutovella aljutovica, A. intermixta, Neostaffella subquadrata and Eofusulina sp.

\section{CONCLUSIONS}

The Bashkirian-Moscovian interval in the Kuzuoluk section has been characterized by lithofacies and fusulinid assemblages and the following conclusions can be drawn.

Sediments yielded rich dasycladacean algae assemblages (mainly Donezella and Beresella) which indicate shallow-marine palaeoenvironments (Wray, 1977; Krainer et al., 2003). The absence of conodont remains and an abundance of fusulinids supports this interpretation (Brasier, 1980).

The foraminiferal faunas recorded can be correlated with the assemblages from Tien-Shan, the Southern Urals and the Russian Platform. Many species from Tien-Shan are also similar to those from Kuzuoluk.
The Kuzuoluk section is characterized by a continuous succession of strata across the Bashkirian-Moscovian boundary which contains abundant and well-preserved fusulinid faunas and is one of the best Carboniferous sections in the Siyah Aladag Nappe in the eastern Taurides. Further studies involving associated microfossils, such as small foraminifera, as well as sequence stratigraphy and magnetostratigraphy may provide additional important datums for determining and correlating the Bashkirian-Moscovian boundary in this section. Moreover, the Kuzuoluk section is one of the best candidate sections for studying the Bashkirian-Moscovian boundary in the Taurides and has potential for regional correlation.

\section{ACKNOWLEDGEMENTS}

This study is a part of MTA projects (16B9) and supported by the General Directorate of Mineral Research and Exploration (MTA). The authors are extremely grateful to A. Neyevin (KMEGEI), D. G. Saydam (MTA), T. Vorobiev (KMEGEI), E. Corekcioglu (MTA), E. Ekmekci (MTA) and B. O. Ozturk (MTA) for their contributions during the different stages of this study. Thanks are also due to U. Kagan Tekin (H.U) for reviewing the first version of the manuscript and suggesting a number of useful changes. The authors gratefully acknowledge J. R. Groves (University of Northern Iowa, USA) and I. D. Somerville (University College Dublin, Ireland) who have kindly reviewed this manuscript and made valuable suggestions.

\section{Manuscript received 16 March 2005 Manuscript accepted 14 January 2007}

\section{REFERENCES}

Altiner, D. 1981. Recherches stratigraphiques et micropaléontologique dans le Taurus oriental au $N W$ de Pinarbasi (Turquie). PhD Thése, 2005. Université de Genéve, 450pp.

Altiner, D. \& Ozgul, N. 2001. Carboniferous and Permian of the allochthonous terrains of the Central Tauride Belt, Southern Turkey. International Conference on Paleozoic Benthic Foraminifera, 25-29 August, Excursion Guide Book. 
Altiner, D. \& Zaninetti, L. 1980. Les Archaediscidae (Foraminifères) dans le Carbonifère inférieur et moyen du Taurus oriental, Turquie. Rivista Italiana di Paleontologia e Stratigrafia, 86 (2): 273-316.

Ayhan, A. \& Lengeranli, Y. 1986. Tectonostratigraphical features of Aladag Region between Yahyali and Demirkazik. Geological Engineering, Bulletin of the Chamber of Geological Engineers of Turkey, 27: 31-45 [in Turkish with English abstract].

Blumenthal, M.M. 1941. Apercu de la Geologie du Taurus dans les Vilayets Nigde et d'Adana. Mineral Research and Exploration Publications, Serie B, 6: 1-48.

Blumenthal, M.M. 1951. Recherches géologiques dans le Taurus occidental dans l'arriére-pays d'Alanya. Mineral Research and Exploration Publications, Serie D, 5: 1-134.

Brasier, M.D. 1980. Microfossils. George Allen \& Unwin, London, 193pp.

Davydov, V.I. 2002. Carboniferous System and the current status of its subdivisions. In: Chuvashov, B.I. (Ed.), Carboniferous Stratigraphy and Paleogeography of Eurasia. Institute of Geology and Geochemistry of Urals Branch of Russian Academy of Sciences, Ekaterinburg, 72-91.

Davydov, V.I., Glenister, B.F., Spinosa, C., Ritter, S.M., Chernykh, V.V., Wardlaw, B.R. \& Synder, W.S. 1998. Proposal of Aidaralash as global stratotype section and point (GSSP) for base of the Permian system. Episodes, 21 (1): 11-17.

Dzhenchuraeva, A.V. 1979. Stratigraphy and foraminifera of the Middle Carboniferous deposits of the northern slopes of Turkestan-Alai. Izdatel'stvo Ilim, Frunze, 100pp. [in Russian].

Dzhenchuraeva, A.V. 1997. Biostratigraphy of Middle and Upper Carboniferous deposits of Tien-Shan. In: Ross, C.A., Ross, J. \& Brenckle, P. (Eds), Late Paleozoic Foraminifera; their biostratigraphy evolution and paleoecology; and the Mid-Carboniferous boundary. Cushman Foundation for Foraminiferal Research, Special Publication, 36: 37-40.

Dzhenchuraeva, A.V. 2000. Settling down of the Bashkirian and Moscovian stages (Middle Carboniferous) in the Southern Tien-Shan. Carboniferous zonal subdivisions of the Russian general stratigraphical scale. Rossiyskaya Akademiye nauk, Ufimskiy nauchnyy tsentr, Institut geologii, Izdatel'stvo 'Gilem', Ufa, 34-36 [in Russian].

Dzhenchuraeva, A.V. 2001. Foraminifera zoning of Bashkirian stage and Lower Moscovian substage in Turkestan-Alai (South Tien-Shan). International Conference on Paleozoic Benthic Foraminifera, 20-24 August, Abstracts: 11.

Groves, J.R. 2003. Early Progress Toward Selecting a GSSP for the Bashkirian-Moscovian Boundary: biostratigraphic and chemostratigraphic considerations. XVth International Congress on Carboniferous and Permian Stratigraphy, 10-16 August, Abstracts: 186.

Guvenc, T. 1965a. Etude stratigraphique et micropaléontologique du Carbonifère et du Permien des Taurus Occidentaux dans l'arriere pays d'Alanya, Turquie. PhD Thése, 3 vols. Université de Paris, 273pp.

Guvenc, T. 1965b. Représentants des Bereselleae (Algues calcaires) dans le Carbonifère de Turquie et description d'un nouveau genre: Goksuella n. g. Bulletin de la Société Géologique de France, 7 (7): 843-850.

Guvenc, T. 1972. Description d'un nouveau genre d'Algues calcaires (Gymnocodiacées et Dasycladacées) du Carbonifère et du Permien des Taurus occidentaux (Turquie). Revue de Micropaléontologie, 9 (2): 94-103.

Guvenc, T. 1977. Stratigraphie du Carbonifère et du Permien de la Nappe de Hadim. 6th Colloquium of the Geology of Aegean Regions. Aegean University, 251-261.

Isakova, T.N. 1998. Settling down of a new scale of the Moscovian Stage on fusulinid fauna, boundary of the stages and biotic events of the Middle and Late Carboniferous. Report Thesis of all-Russian Meeting, 7-13 August, Moscow: 24-25 [in Russian].
Isakova, T.N. 2001. Chapter 1. Fusulinida. In: Alekseev, A.S. \& Shik, S.M. (Eds), Middle Carboniferous of Moscow Syneclise (southern part), Volume 2, Biostratigraphy. Scientific World, Moscow, 10-32 [in Russian].

Ivanova, R.M. 2000. New species of foraminifera from Bashkirian stage deposits (Carboniferous) of the Southern and Middle Urals. Materials on stratigraphy and paleontology of the Urals. Izdatel'stvo, Urals Branch of Russian Academy of Science, 4: 39-44 [in Russian].

Ivanova, R.M. 2002. Fusulinid zones of the Moscovian Stage in Urals. Carboniferous stratigraphy and paleogeography in Eurasia. Institute of Geology and Geochemistry of UBRAS, 00: 127-138 [in Russian].

Krainer, K., Vachard, D. \& Spencer, G.L. 2003. Microfacies and microfossil assemblages (smaller Foraminifers, algae, pseudoalgae) of the Hueco Group and Laborcita Formation (Upper PennsylvanianLower Permian), south-central New Mexico, USA. Rivista Italiana di Paleontologia e Stratigrafia, 109 (1): 3-36.

Kulagine, E.I., Pazukhin, V.N., Kochetkova, N.M., Sinitsyna, Z.A. \& Kochetova, N.N. 2001. The stratotype and key section of the Bashkirian Stage (Carboniferous) in the Southern Urals. Russian Academy of Sciences, Ufa Research Center, Institute of Geology, Upravlenie po Nedram Respubliki Bashkortostan, Akademiya Nauk Respubliki Bashkortostan, Otdelenie Nauk o Zemle (Ufa), 139pp.

Lane, H.R., Brenckle, P.L., Baesemann, J.F. \& Richards, B. 1999. The IUGS boundary in the middle of the Carboniferous: Arrow Canyon, Nevada, USA. Episodes, 22 (4): 272-283.

Okuyucu, C. 2001. Late Moscovian and Kasimovian fusulinid fauna of the Ozbek Tepe Section, Eastern Taurides, Turkey. International Conference on Paleozoic Benthic Foraminifera, 20-24 August, Abstracts: 34

Okuyucu, C. 2002. Micropaleotological study of the CarboniferousPermian passage of Anatolian Platform in Taurus Mountains. PhD Thesis, Hacettepe University, Ankara, 207pp. (in Turkish).

Okuyucu, C. 2003. Late Moscovian-middle Kasimovian fusulinid fauna from the Anatolian Platform, eastern and central Taurides, Southern Turkey. XVth International Congress on Carboniferous and Permian Stratigraphy, 10-16 August, Abstracts: 378.

Ozgul, N. 1976. Some geological aspects of the Taurus orogenic belt. Geological Society of Turkey Bulletin, 19: 65-78 [in Turkish with English abstract].

Ozgul, N. 1984. Stratigraphy and tectonic evolution of the Central Taurides. In: Tekeli, O. \& Goncuoglu, M.C. (Eds), Geology of the Taurus Belt. Publication of General Directorate of Mineral Research and Exploration, Ankara, 77-90.

Ozgul, N. 1997. Stratigraphy of the tectono-stratigraphic units in the region Bozkir-Hadim-Taskent (north central Taurides). Mineral Research and Exploration Institute of Turkey Bulletin, 119: 113-174 [in Turkish with English abstract]

Paproth, E., Feist, R. \& Flajs, G. 1991. Decision on the DevonianCarboniferous boundary stratotype. Episodes, 14 (4): 331-336.

Rumyantseva, Z.S. 1974. Stratigraphy and foraminifers of the Middle Carboniferous of the Central Kyzyl-Kum. Izdatel'stvo Fan Uzbekskoi SSR, 138pp.

Solovieva, M.N. 1977. Zonal stratigraphy of the Middle Carboniferous based on the fauna of the Fusulinacea. Voprosy Mikropaleontologii, 19: 43-64 [in Russian].

Solovieva, M.N. 1986. Fusulinid zonation of the Moscovian Stage as a result of restudying stratotypes of the intrastage units. Voprosy Mikropaleontologii, 28: 3-23 [in Russian].

Villa, E. 1995. Fusulinaceos Carboniferos del este de Asturias ( $N$ de Espana). Biostratigraphie du Paléozoique, 13. Université Claude Bernard Lyon, 261pp.

Wray, J.L. 1977. Calcareous Algae. Developments in Paleontology and Stratigraphy, 4. Elsevier, Amsterdam, 185pp. 